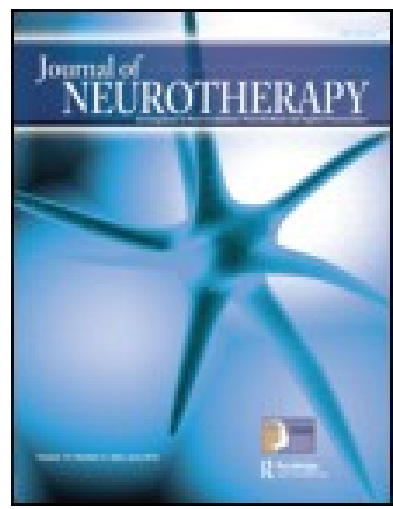

J ournal of Neurotherapy: Investigations in Neuromodulation, Neurofeedback and Applied Neuroscience

\title{
Pilot Data Assessing the Functional Integrity of the Default Network in Adult ADHD with fMRI and sLORETA
}

\author{
Rex Cannon ${ }^{a b}$, Cynthia Kerson ${ }^{c}$, Adam Hampshire ${ }^{d} \&$ Coleman L. Garner ${ }^{a}$ \\ ${ }^{a}$ Clinical Neuroscience, Self-Regulation and Biological Psychology Laboratory, Department of \\ Psychology, University of Tennessee, Knoxville, Knoxville, Tennessee, USA \\ ${ }^{b}$ Cole Neuroscience Center: Memory Disorders Clinic, University of Tennessee Graduate \\ School of Medicine, Knoxville, Tennessee, USA \\ c ISNR Research Foundation, San Rafael , California, USA \\ ${ }^{\mathrm{d}}$ Centre for Brain and Mind, Department of Psychology, University of Western Ontario , \\ London, Ontario, Canada \\ Published online: 21 Nov 2012.
}

To cite this article: Rex Cannon, Cynthia Kerson, Adam Hampshire \& Coleman L. Garner (2012) Pilot Data Assessing the Functional Integrity of the Default Network in Adult ADHD with fMRI and sLORETA, J ournal of Neurotherapy: Investigations in Neuromodulation, Neurofeedback and Applied Neuroscience, 16:4, 246-263, DOI: 10.1080/ 10874208.2012.730408

To link to this article: http:// dx.doi.org/ 10.1080/ 10874208.2012.730408

PLEASE SCROLL DOWN FOR ARTICLE

(c) International Society for Neurofeedback and Research (ISNR), all rights reserved. This article (the "Article") may be accessed online from ISNR at no charge. The Article may be viewed online, stored in electronic or physical form, or archived for research, teaching, and private study purposes. The Article may be archived in public libraries or university libraries at the direction of said public library or university library. Any other reproduction of the Article for redistribution, sale, resale, Ioan, sublicensing, systematic supply, or other distribution, including both physical and electronic reproduction for such purposes, is expressly forbidden. Preparing or reproducing derivative works of this article is expressly forbidden. ISNR makes no representation or warranty as to the accuracy or completeness of any content in the Article. From 1995 to 2013 the J ournal of Neurotherapy was the official publication of ISNR (www. Isnr. org); on April 27, 2016 ISNR acquired the journal from Taylor \& Francis Group, LLC. In 2014, ISNR established its official open-access journal NeuroRegulation (ISSN: 2373-0587; www. neuroregulation. org).

THIS OPEN-ACCESS CONTENT MADE POSSIBLE BY THESE GENEROUS SPONSORS

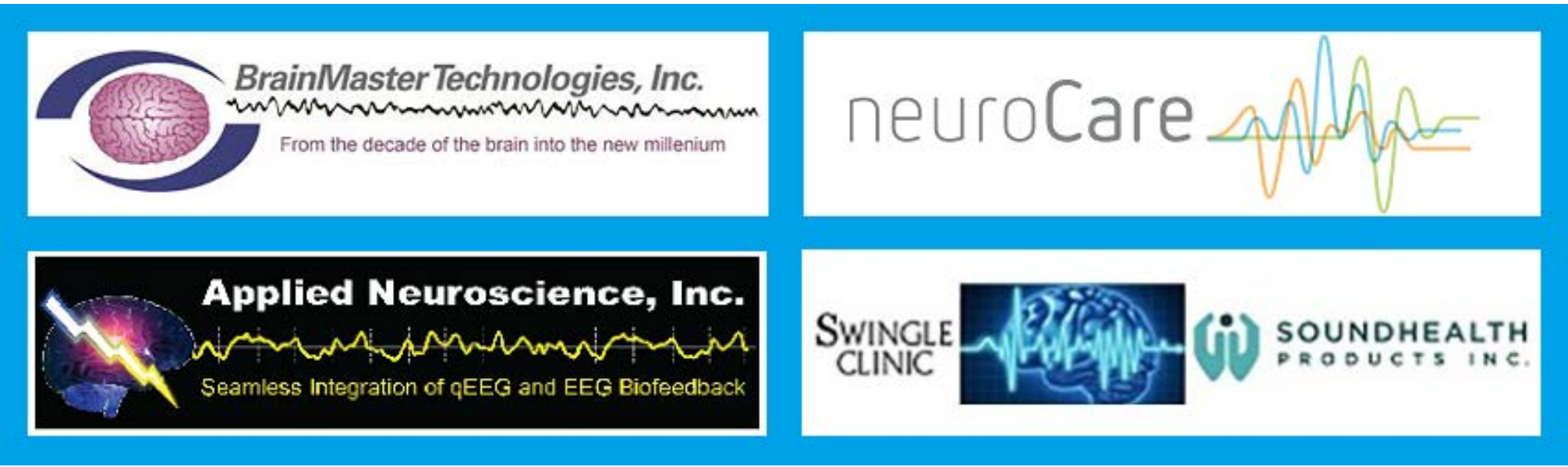




\title{
PILOT DATA ASSESSING THE FUNCTIONAL INTEGRITY OF THE DEFAULT NETWORK IN ADULT ADHD WITH fMRI AND sLORETA
}

\author{
Rex Cannon $^{1,2}$, Cynthia Kerson ${ }^{3}$, Adam Hampshire ${ }^{4}$, Coleman L. Garner ${ }^{1}$ \\ ${ }^{1}$ Clinical Neuroscience, Self-Regulation and Biological Psychology Laboratory, Department \\ of Psychology, University of Tennessee, Knoxville, Knoxville, Tennessee, USA \\ ${ }^{2}$ Cole Neuroscience Center: Memory Disorders Clinic, University of Tennessee Graduate School \\ of Medicine, Knoxville, Tennessee, USA \\ ${ }^{3}$ ISNR Research Foundation, San Rafael, California, USA \\ ${ }^{4}$ Centre for Brain and Mind, Department of Psychology, University of Western Ontario, London, \\ Ontario, Canada
}

Intrinsic functional connectivity within the default mode network (DMN) of the brain has gained growing interest in attention deficit/hyperactivity disorder (ADHD). The DMN is proposed to support such core functions as theory of mind, self-related activities such as autobiographical self, stimulus independent thought, self-projection, self-reference, and introspective processes as well as central features of self-regulation, task compliance, and executive functions. Based on prior data showing that sLORETA and FMRI localize DMN regions with complementary accuracy, we hypothesized that this combination of methods could provide important information about the functional integrity of DMN connectivity with special attention between parietal and medial prefrontal regions. This study was conducted with 6 adults with ADHD and 7 age-similar, nonclinical controls. The present study recorded brain activity using both EEG and fMRI during rest and a Stroop paradigm. For this study only the eyes-opened rest data were analyzed using the sLORETA and FMRI psychophysiological interaction model respectively. sLORETA functional connectivity differences were assessed with Pearson's correlation measures and Fisher's $Z$ test to examine the strength of the obtained coefficients between groups within the DMN. Differences in functional integrity of the DMN were found for most frequencies except theta, which did not show any difference between groups. Of interest, the ADHD group showed greater intrinsic functional connectivity in the alpha frequency range between medial prefrontal and left parietal regions. Functional connectivity within the DMN in both groups was comparable between both imaging modalities, with sLORETA providing evidence of the frequency-specific meaning of the associations. This study furthers the requisite for utilizing sLORETA in combination with FMRI to obtain knowledge of the connectivity in the context of EEG frequency-specific parameters. The study data offer preliminary evidence demonstrating the importance of left parieto-occipital regions to ADHD and potential neural resources that may be compromised in ADHD that are specifically involved in self-regulation, working memory, and executive functions.

Received 18 May 2012; accepted 22 August 2012.

This study was partially funded by the ISNR Research Foundation, the Hubbard Foundation, Mitsar, Ltd., and NovaTech EEG. The authors have no conflicts of interest.

Address correspondence to Cynthia Kerson, PhD, ISNR Research Foundation, 1925 Francisco Boulevard E. \#12, San Rafael, CA 94901, USA. E-mail: executivedirector@isnr-researchfoundation.org 


\section{INTRODUCTION}

Intrinsic functional connectivity within the default mode network (DMN) of the brain has gained growing interest in attention deficit/hyperactivity disorder (ADHD; Tian et al., 2008; Zang et al., 2007). Functional connectivity (FC) analyses typically examine the correlations between neural regions that may or may not share known neuroanatomical connections (Smith et al., 2009) conducted utilizing functional magnetic resonance imaging (fMRI) and the electroencephalogram (EEG) with connectivity measures of coherence and phase-lag. In many cases the correlations or partial correlations between brain regions during baseline or specific tasks are measured and the change in strength (or degree) of the correlation is utilized to evaluate the differences in resting networks relative to task (Brookes et al., 2011; Cannon, Congedo, Lubar, \& Hutchens, 2009; Cannon et al., 2007; Fair et al., 2008; Sun et al., 2012). The DMN is one of the more widely investigated networks associated with baseline or resting conditions and consists of functionally related regions (Table 1) that consistently show increased activity during rest with the eyes closed as compared to functionally specific cognitive tasks and/or the eyes-opened resting condition (Shulman et al., 1997; Shulman et al., 1999; Shulman, Schwarz, Miezin, \& Petersen, 1998). It has also been noted that attention lapses have been found to occur shortly after periods of decreased deactivation of posterior DMN regions (Weissman, Roberts, Visscher, \& Woldorff, 2006). The DMN is synonymous with the resting state network (RSN); however, the RSN has been suggested to include numerous networks of functionally connected neuronal assemblies (Damoiseaux et al., 2008; Damoiseaux et al., 2006; Fransson et al., 2007). It is important to note that the DMN is not to be confused with networks of executive attention or networks associated with monitoring attention and the physical body (Buckner, Andrews-Hanna, \& Schacter, 2008). However, the complex relationships between differential networks and their respective assemblages are of interest in both normal and clinical populations.

The DMN and other network assemblages are important to the study of ADHD brain function due to the intricate relations between networks and their inclusive functional components (e.g., FC between network hubs and nodes). The primary components of the DMN comprise medial (medial prefrontal cortex, posterior cingulate/precuneus) and lateral (posterior parietal) brain regions that routinely exhibit decreases in coherent activity during attention-demanding cognitive tasks (Raichle et al., 2001). The relevance of the DMN to ADHD indicates that a failure for the DMN to decrease in power and connectivity when the eyes are opened or during an attention or

TABLE 1. Default Network Regions Used in This Study

\begin{tabular}{lrrll}
\hline Orientation Brodmann area & X & Y & Z & Neuroanatomical label \\
\hline (1) Medial 31/7 & -5 & -49 & 40 & Dorsal posterior cingulate/Precuneus \\
(2) Left 40 & -53 & -39 & 42 & Parietal lobe/Supramarginal gyrus \\
(3) Left 39/19 & -45 & -67 & 36 & Angular gyrus \\
(4) Right 40 & 45 & -57 & 34 & Parietal lobe/Supramarginal gyrus \\
(5) Left lateral 8 & -27 & 27 & 40 & Frontal eye fields \\
(6) Right 8/9 & 5 & 49 & 36 & Frontal lobes \\
(7) Left 9 & -15 & 55 & 26 & Dorsolateral prefrontal cortex \\
(8) Left 10 & -19 & -1 & -4 & Anterior prefrontal cortex \\
(9) Medial 10 & -1 & -6 & Middle frontal lobe \\
(10) Left 10/47 & -33 & 35 & -10 & Anferior frontal lobe \\
(11) Medial 32 & 3 & -19 & -18 & Inferior cingulate \\
(12) Left 20 & -49 & &
\end{tabular}

Note. From left to right are the Brodmann Area and orientation in the brain, the $x, y$, and $z$ coordinates in MNI space and the neuroanatomical label. 
cognitive task, as is shown in normal populations, reflects disruptions in both attention networks and self-regulatory mechanisms (Cannon, Baldwin, DiLoreto, \& Khadoumma 2011; Castellanos et al., 2002; Weissman et al., 2006). These connectivity issues are important to ADHD for several reasons: Structural MRI studies have shown a consistent reduction in cerebral volume in several regions of the brain in children and adults, including the right hemisphere (Castellanos et al., 2002), frontal and occipital regions (Castellanos \& Acosta, 2002), and total white matter volumes in the left occipital region. This particular region in the left occipital cortex shows similar white matter volume deficits in unaffected siblings, suggesting a potential marker for increased familial risk for ADHD (Durston et al., 2004). Numerous studies have shown cerebral volume reduction in ADHD in a variety of regions; however, procedural issues regarding methodology influence the effectiveness of conclusions or robust agreement about specific etiology (van Ewijk, Heslenfeld, Zwiers, Buitelaar, \& Oosterlaan, 2012). There have been reports of negligible volume reductions in ADHD (Wolosin, Richardson, Hennessey, Denckla, \& Mostofsky, 2009). Investigations of white matter volume in ADHD consistently report a reduction in ADHD patients as compared to controls across the entire cerebral volume (Castellanos et al., 2002; D'Agati, Casarelli, Pitzianti, \& Pasini, 2010; McAlonan et al., 2007). ADHD is a diverse developmental condition with various potential loci of neural dysfunction. Castellanos et al. (2008) also showed decreased functional connectivity between the precuneus and other DMN regions in adults with ADHD and decreased DMN network homogeneity in the precuneus in ADHD as compared to controls. The precuneus is a prominent node in the DMN that has been receiving increasing attention in the ADHD neuroimaging literature, showing reductions in grey matter volume in ADHD samples (Carmona et al., 2005; Overmeyer et al., 2001). The precuneus has been shown to play an important role in cognitive and self-relevant processing (Enzi, de Greck,
Prosch, Tempelmann, \& Northoff, 2009; Northoff, 2005; Northoff \& Bermpohl, 2004; Northoff et al., 2006; Northoff et al., 2009). It may also play an important function in affect and self-regulatory functions given its widespread connectivity with the anterior cingulate, lateral prefrontal cortex, and inferior and superior parietal lobes and subcortical connections including the thalamus, striatum, and brainstem regions (Cavanna \& Trimble, 2006).

In recent years there has been a growing interest in mapping out structural connectivity of the brain with diffusion tensor imaging (DTI) in ADHD (van Ewijk et al., 2012). DTI is an MRI-derived technology that models the local diffusion of water as a function of direction. This distribution is determined by the microscopic resistance of water molecules when diffusing through tissue (Basser, Mattiello, \& LeBihan, 1994a, 1994b). DTI has been utilized to investigate structural white matter connectivity between several putative cerebral structures thought to be instrumental mechanisms in ADHD. For example, ADHD patients showed decreased fractional anisotropy in the isthmus of the corpus callosum (CC) with no differences found in any other regions of the CC (Cao et al., 2010). Similar negative findings in the $\mathrm{CC}$ were also reported by Hamilton et al. (2008). Another study focused on the basal ganglia and its associated structures that are thought to play a primary role in ADHD, including the caudate, putamen/globus pallidus, and thalamus (Silk, Vance, Rinehart, Bradshaw, \& Cunnington, 2009). Notably, no differences were found between $\mathrm{ADHD}$ and controls in these regions of interest (ROls). However, differences were found in developmental trajectories (path, progression, and line of development) that mainly involved the caudate nucleus. Other regions that have been investigated with null or minimal differences include the cerebellum (Bechtel et al., 2009), superior longitudinal fasciculus and splenium of the CC (Pavuluri et al., 2009; Takahashi et al., 2010) and left sagittal stratum (Peterson et al., 2011). A more recent study in adult ADHD reported microstructural differences in the isthmus and splenium 
without macrostructural differences seen in the CC (Dramsdahl, Westerhausen, Haavik, Hugdahl, \& Plessen, 2012). Valdes-Hernandez and colleagues (2010) reported an important translational finding relating EEG spectral properties with white matter architecture (Valdes-Hernandez et al., 2010). They demonstrated that posterior commissural fibers within the $\mathrm{CC}$ showed the strongest correlation with the alpha EEG frequency $(8-12 \mathrm{~Hz})$, such that the Isthmus and Tapetum in the superior occipital cortex showed the highest positive association with the spectral peak of the resting alpha frequency in study subjects. These relationships are suggested to represent corticothalamocortical cycles that may modulate the alpha frequency domain. It is interesting that white matter architecture is associated more with the alpha frequency domain than with the neocortical region or grey matter (Valdes-Hernandez et al., 2010). Therefore, the combined DTI, structural MRI of both grey matter and white matter volumes in left parieto-occipital regions, functional connectivity in the DMN regions and functional connectivity between these parieto-occipital and medial prefrontal regions are of profound interest to our research group.

ADHD has been studied extensively using quantitative electroencephalography (qEEG) (Arns, Drinkenburg, \& Leon Kenemans, 2012; Castellanos et al., 1996; Ogrim, Kropotov, \& Hestad, 2012). QEEG is the noninvasive procedure in which the brain's electrical processes are recorded in digitized format utilizing computerized imaging and statistical procedures. The oscillatory activity in the qEEG is thought to reflect frequency specific networks in the brain (Engel, Fries, \& Singer, 2001). EEG is a direct and reliable signature of neural activity and provides ideal temporal resolution in the millisecond time domain (Cannon et al., 2012; Coburn et al., 2006; Hughes \& John, 1999). Notably, Mazaheri and colleagues examined functional disconnection between frontal and visual cortices in typical children and those with ADHD in a cross-modal attention task. They found that alpha activity was differentially modulated and inversely correlated with mid-frontal theta activity (Mazaheri et al., 2010) in normal children but not in ADHD. The authors concluded that the decrease in alpha shown in typical children was associated with top-down control of attentional systems by frontal theta and children with ADHD did not exhibit cue-induced posterior alpha reductions even though this could not be attributed to failure to perform the task, as the ADHD children were slower than normal controls but did complete the task (Mazaheri et al., 2010). These types of research findings are important given the search for specific biomarkers to more accurately diagnose ADHD as well as mechanisms to determine successful treatment outcomes (e.g., does the brain change with temporal synchrony in performance on testing procedures?). These are important directions for future study.

Standardized low-resolution brain electromagnetic tomography (sLORETA) is a method of probabilistic source estimation of EEG signals in standardized brain atlas space utilizing a restricted inverse solution (Pascual-Marqui, Michel, \& Lehmann, 1994; Pascual-Marqui, Esslen, Kochi, \& Lehmann, 2002). sLORETA has been used to examine EEG sources in depression (Pizzagalli, Oakes, \& Davidson, 2003), epilepsy (Zumsteg, Andrade, \& Wennberg, 2006), and evaluating temporal changes associated with differential task specific default network activity (Cannon \& Baldwin, 2012). Recently, sLORETA and fMRI were shown to localize DMN regions with complementary accuracy (Cannon, Kerson, \& Hampshire, 2011). This study was conducted with one apriori hypothesis and two exploratory aims. First, given the indications of Castellanos et al. (2008) and Mazaheri et al. (2010), we hypothesized that we would find differences in the degree of functional connectivity in the DMN between ADHD and controls. Next, we sought to examine connectivity between parieto-occipital and medial prefrontal regions, with special attention directed toward alpha and theta frequency domains between individuals with ADHD and controls. Finally, we sought to explore the utility of consecutive measurements using fMRI and 
sLORETA for improving our understanding of the relationship and meaning of the EEG and FMRI in the DMN (Cannon \& Baldwin, 2012; Cannon, Kerson, et al. 2011).

\section{METHODS}

Twenty-four potential subjects were recruited through local practitioners and children and adults with ADHD. Of these, 10 non-ADHD and nine ADHD subjects were recruited. Of the five who were excluded, three reported current major depressive disorder and were on selective serotonin reuptake inhibitors, one reported recent history of a suicide attempt, and one did not want to participate without pay. However, three non-ADHD recruited subjects did not complete the study (one became anxious in the MRI scanner, one cancelled due to time constraints, and one was physically unsuited), and three recruited ADHD subjects were disqualified (two became anxious in the scanner and the third rescinded consent after completing both the $\mathrm{FMRI}$ and EEG sections of the evaluation). Therefore, this study was conducted with 13 total participants - seven nonclinical controls and six adults with a standing diagnosis of ADHD. All participants in this study were right-handed. After signing the informed consent, each participant first underwent a qEEG or fMRI scan, depending upon a random assignment, and then proceeded to the respective imaging or qEEG device.

Subjects were interviewed via phone or Skype following a script that ruled out suicidal ideation, traumatic brain injury, medication intake other than stimulants (which were ceased 3 days prior to the examination), claustrophobia, pregnancy, nonambulatory access, schizophrenia or other Axis III disorder, and metal implants; each subject verbally responded to the Mini International Neuropsychiatric Interview questionnaire for inclusion into the ADHD or non-ADHD group. The participants also completed the Conners' Adult ADHD Rating Scale-Self-Report: Short Version (CAARS-S:S; Conners et al., 1999). The participants were screened using a questionnaire specific to ADHD symptomatology during the initial interview. The answer scale is never, rarely, sometimes, often, and very often. Should the subject answer mostly often or very often to these six questions, he or she would be admitted into the study as an ADHD subject (following congruent results on the CAARS-S:S). Conversely, those who answered mostly never or rarely would be included in the control group. The verbally administered Mini International Neuropsychiatric Interview instrument was intended to rule out comorbid disorders, including major depressive disorder, mania, panic disorder, obsessive-compulsive disorder, social phobia, psychotic disorders including schizophrenia, posttraumatic stress disorder, anorexia or bulimia, antisocial personality disorder, and other mood disorder with psychotic features. Alcohol or psychoactive substance abuse or dependence is also usually questioned during this interview, but we did not include those questions, as they posed ethical issues. Two ADHD subjects reported history of mild depression and one reported anxiety, although neither was medicated. We accepted these subjects because they were not medicated and symptoms were not current. None of the controls reported symptoms from any of the aforementioned disorders during this interview. There are 26 questions on the CAARS-S:S that distinguish non-ADHD adults from ADHD adults. The answer scale is 0 (not at all, never), 1 (just a little, once in a while), 2 (pretty much, often), and 3 (very much, very frequently), and the answers that result in scaling for inattention are: memory problems, hyperactivity, restlessness, impulsivity and emotional lability, problems with self-concept, and an overall ADHD index.

The six ADHD adults in this study had an average of 21.5 on the overall ADHD index (92 percentile; raw score was 22.33 for the women [97 percentile] and 20.66 for the men [87 percentile]). This is contrasted by the average overall ADHD index of 10.5 for controls (41.14 percentile; 10.4 for the women [52 percentile] and 7 for the men [25.66 percentile]). 


\section{Procedures}

The subjects were seated in a quiet room away from any telephones and other ordinary office noise. Their scalps were prepped with NuPrep and alcohol to clean and abrade the skin at the 19 electrode sites as designated by the 10/20 international system (Jasper, 1958) and the linked-ear reference sites. The subjects were then fitted with a spandex cap (ElectroCap International, Eaton, $\mathrm{OH}$ ) that contains electrodes at the 10/20 sites, aligning the electrodes to the proper locations on the scalp. The subjects were briefly educated on artifact production and contamination (e.g., how the signal compared when calm and relaxed to during eye blinks and saccades and when shoulder, facial, and or neck tension persisted) and asked to remain quiet and still during the recording session. The participants completed 5-min eyes-closed and eyes-opened resting baselines. Event-related potential procedures were also conducted using the Stroop task; however, these results are not presented in this work.

EEG recordings were acquired using the Mitsar 202 (Mitsar Ltd, Saint Petersburg, Russia) amplifier system. The EEG was sampled by 24-bit AD converter at 250 samples per second, and the low and high pass filters were set at 0.0 and $50.0 \mathrm{~Hz}$, respectively. Data were acquired using the WinEEG acquisition program. Data were then transported into the NovaTech EureKa! EEG editing software (Congedo, Ozen, \& Sherlin, 2002), in which it was plotted and carefully inspected using manual artifact rejection. All episodic artifacts including eye blinks, eye movements, teeth clenching, body movements, or EKG artifact were removed from the EEG stream.

The fMRI scanner was a Siemens 3 T Tim Trio. The images were collected every $2 \mathrm{~s}$. The scanner employed Syngo B15 software with the BOLD fMRI and Neuro3D packages. The RF transmitter contains one $35 \mathrm{~kW}$ narrowband RF transmitter and one $8 \mathrm{~kW}$ broadband RF transmitter. Its gradient strength/slew strength is $\leq \mathrm{mTIM} / \leq 200 \mathrm{~T} /\left(\mathrm{m}^{*} 2\right)$ with 32 receiver channels. The stimuli delivery system was an Avotec Silent Scan 3100 research audio system and NEC NP 4000, XGA $1280 \times 768$ rear projection system. A luminit 75-degree diffuse screen and Presentation software were used for the task presentation. A fiber optic keypad was used for participant responses. All parameters for data collection can be found in Cannon, Kerson, et al. (2011). Subjects were instructed about the tasks they were to perform in the scanner. In this case, the FANTAB Neuropsychological Battery was used, which is a version of the CANTAB (Cambridge, England) Neuropsychological Test Battery that is fashioned to work in the scanner. After task instruction, the subject changed into hospital scrubs. They were covered with a blanket and fitted for the head-motion cage. The keypad was positioned to their dominant hand. They were shuttled into the scanner, given time to acclimate, and began with cross-hair fixation to be used as the baseline comparator to the tasks, followed by the FANTAB battery. At the end they remained still, listening to music for $12 \mathrm{~min}$ to record the individual anatomical aspects of their brain. A T1-weighted anatomical image was also acquired for registration purposes $(\mathrm{MP}-\mathrm{RAGE}, \mathrm{TR}=2500 \mathrm{~ms}$; $\mathrm{TE}=4.35 \mathrm{~ms}$; $\mathrm{TI}=900 \mathrm{~ms} ; \quad$ Flip angle $=8 ; 176$ slices; $\mathrm{FOV}=256 \mathrm{~mm}$ ).

\section{fMRI Data Analysis}

FMRI data were preprocessed using the SPM 2005 software package (Wellcome Department of Cognitive Neurology; http://www.fil. ion.ucl.ac.uk/spm/). Time-courses were motion and slice-time corrected, normalized to the standard MNI template, smoothed with an $8 \mathrm{~mm}$ Gaussian kernel, and high-pass filtered prior to analysis (cutoff 180s). Individual subjects' data were modeled in SPM5 using the general linear model. Four predictor functions were included in the model corresponding to the onsets and durations of the four task event types convolved with the standard hemodynamic response function. Rotational and translational movement parameters within the $x, y$ and $z$ planes were included as an additional six regressors. Blocks of rest were included in 
the task design, and these allowed the task regressors to be calculated relative to a constant that included resting baseline activity. Group-level analyses were carried out using the MarsBaR ROI toolbox for SPM, which calculates the average level of activation across all voxels within predefined ROls.

\section{sLORETA Data Analysis}

The EEG stream was edited using EureKa 3 software (NovaTech EEG, Mesa, AZ). EEG resampling was obtained by means of natural cubic spline interpolation, as the EEG is a continuous signal constituted by oscillation of potential differences over time (Congedo et al., 2002). All active task conditions and baseline data were processed with particular attention given to the frontal and temporal leads. All episodic eye blinks, eye movements, teeth clenching, jaw tension, body or neck movements, and possible electrocardiogram (EKG) were removed from the EEG stream. Fourier cross-spectral matrices were then computed and averaged over $75 \%$ overlapping 4 -s artifact-free epochs, which resulted in one cross-spectral matrix for each subject for each discrete frequency. The EEG data were analyzed utilizing the following frequency domains: Delta $(0.5-3.5 \mathrm{~Hz})$; Theta $(3.5-$ $7.5 \mathrm{~Hz}) ; \quad$ Alpha 1 (7.5-10.0 Hz); Alpha 2 $(10.0-12.0 \mathrm{~Hz})$, and Beta $(12.0-32.0 \mathrm{~Hz})$.

\section{sLORETA Functional Connectivity}

The sLORETA functional connectivity analyses involved three steps. First, we created an ROI file with the MNI coordinates for the 12 seed points of the DMN regions (see Table 1). Each of these ROI values consisted of the current source density (CSD) levels from each ROI seed and one single voxel (its nearest neighbor) for total voxel size $10 \mathrm{~mm}^{3}$. The resulting file produced log transformed average CSD across multiple EEG segments for all subjects for each seed (ROI). The data for these apriori DMN ROIs were organized into Microsoft Excel spreadsheets and then entered into SPSS 19 for analysis. sLORETA images corresponding to the estimated neuronal generators of brain activity within each given frequency range were calculated (Frei et al., 2001). This procedure resulted in one 3D sLORETA image for each subject for each frequency range. The significance threshold was based on a randomization test utilizing 5,000 data randomizations. Second, for each group we took the ROI values for each individual frequency from each ROI utilizing within group correlation analyses. Hence, for each frequency we compared 12 ROI $\times 1$ frequency for seven and six subjects, respectively. Finally, we entered the obtained correlation coefficient for each group for the respective $\mathrm{ROI} \times$ frequency into a Fisher's $Z$ test to test the strength of the correlation (functional connectivity) between groups in regions within the DMN for each frequency domain.

\section{fMRI Functional Connectivity}

We generated a psycho-physiological interaction (PPI) model for each individual in the following manner using the SPM5 software package. Given indications from our previous work (Cannon, Kerson, et al., 2011) showing that medial BA 10 showed significant difference in activation between ADHD and control groups, we extracted the time-course from medial Brodmann area (BA) 10 region of interest (ROI 9 in Table 1) using the SPM VOI function, which pulls out the first eigenvector (principal component) of all voxels within a sphere across time $(10 \mathrm{~mm})$ based at the ROI coordinates. We then generated a psychological function based on rest versus task. More specifically, rest periods were represented with the value 1 and task with the value -1 . The VOI time-course was subjected to a deconvolution with the standard haemodynamic response function (HRF) to generate the likely pattern of underlying neural activity. A psychological interaction was generated by multiplying the psychological and deconvolved medial BA10 time-courses. The PPI timecourse was convolved with the HRF to generate a predictor function. A general linear model was constructed in which activation at each voxel in the brain was fitted with 10 predictors. These predictors were the convolved PPI function, the BA10 time-course, the psychological function convolved with the HRF, 
$6 \times$ movement parameters and a constant term. Whole brain maps depicting beta weights for the first two functions were collected and subjected to group-level random effects analysis.

\section{Alpha-Theta Intercorrelations}

Based on the results from both the sLORETA and FMRI functional connectivity analyses, we also ran correlation procedures between the theta, alpha-1, and alpha-2 frequencies between anterior cingulate (BA 32 ROI 11), medial prefrontal (BA 10 ROI 9), and left parietal (BA 39/19 ROI 3) areas to determine the relationship and visualize differences between alpha and theta bands within and between these cingulate-parietal-medial prefrontal connections for ADHD $(d f=6)$ and controls $(d f=7)$.

\section{RESULTS}

The statistical differences of interarea connectivity between ADHD and control groups show complementary patterns in the $\mathrm{fMRI}$ and sLORETA maps.

\section{SLORETA}

Table 2 summarizes the statistically significant differences of sLORETA connectivity between the two groups. Controls show significantly higher positive associations in the delta and beta frequency domains, except for the associations between the posterior cingulate (BA $31 / 7$ ) and right parietal (BA 40) cortices. Controls also show significantly higher associations between BA 31/7 and left and medial prefrontal cortices than ADHD in the alpha 1 and beta frequencies, whereas ADHD show greater associations in alpha 1 and alpha 2 frequency between left parietal (BA 40 and $39 / 19$ ) and left prefrontal and temporal regions as well as medial BA 32 at the anterior cingulate gyrus. Figure 1 shows the results for the sLORETA connectivity for ADHD > control, control, as found in Table 2. Of interest, there are increased associations for the alpha 1 frequency between left inferior and medial prefrontal cortices for the control group.
TABLE 2. Fisher's $Z$ for sLORETA Connectivity Within the DMN Between Groups in Eyes-Opened Resting Condition

\begin{tabular}{|c|c|c|}
\hline Frequency & Z & p \\
\hline \multicolumn{3}{|l|}{ Delta } \\
\hline R1-R4 & $(-1.71)$ & 0.08 \\
\hline R5-R11 & 1.82 & 0.06 \\
\hline R6-11 & 1.89 & 0.06 \\
\hline R7-R11 & 1.95 & 0.05 \\
\hline R8-R11 & 1.9 & 0.03 \\
\hline R10-R11 & 2.06 & 0.03 \\
\hline R10-R12 & 1.65 & 0.09 \\
\hline \multicolumn{3}{|l|}{ Alpha 1} \\
\hline R1-R8 & 1.74 & 0.08 \\
\hline R1-R9 & 2.17 & 0.02 \\
\hline R1-R10 & 1.8 & 0.07 \\
\hline R1-R12 & 2.16 & 0.03 \\
\hline R2-R12 & $(-1.87)$ & 0.06 \\
\hline R3-R11 & $(-2.11)$ & 0.03 \\
\hline R8-R9 & $(-2.79)$ & 0.005 \\
\hline R9-R10 & -2.15 & 0.03 \\
\hline \multicolumn{3}{|l|}{ Alpha 2} \\
\hline R2-R3 & $(-1.86)$ & 0.06 \\
\hline $\mathrm{R} 2-\mathrm{R} 4$ & $(-1.77)$ & 0.07 \\
\hline R2-R5 & $(-2.04)$ & 0.04 \\
\hline R2-R7 & $(-1.82)$ & 0.06 \\
\hline R2-R8 & $(-2.39)$ & 0.01 \\
\hline R2-R10 & $(-2.75)$ & 0.005 \\
\hline R2-R11 & $(-1.94)$ & 0.05 \\
\hline \multicolumn{3}{|l|}{ Beta } \\
\hline R1-R6 & 2.05 & 0.04 \\
\hline R1-R7 & 1.82 & 0.06 \\
\hline R1-R9 & 2.72 & 0.006 \\
\hline R2-R10 & 2.29 & 0.02 \\
\hline R2-R12 & 2.22 & 0.02 \\
\hline
\end{tabular}

Note. Parenthesis indicates attention deficit/hyperactivity disorder $>$ control. From left to right and top to bottom are the frequency, $Z$, and $p$ value for the delta, alpha- 1 , alpha-2, and beta frequency domains. The specific connectivity contrasts for default mode network (DMN) region of interest pairs showing significant or near significant results are shown for each of the frequency domains. No difference between groups was found in the theta frequency.

Although alpha 1 shows higher connectivity at some regions for the ADHD group and the opposite (control $>$ ADHD) for other regions, alpha 2 shows a specific effect for the ADHD as left BA 39/19 shows greater associations to bilateral prefrontal regions (BA 8, 9, and 10), bilateral parietal cortices and medial prefrontal and AC. On the contrary, the ADHD group shows less connectivity for the alpha 1 and beta frequencies between left BA 31/7, BA 40 , and medial, inferior, and dorsolateral prefrontal as well as inferior fronto-temporal regions within the left hemisphere. 


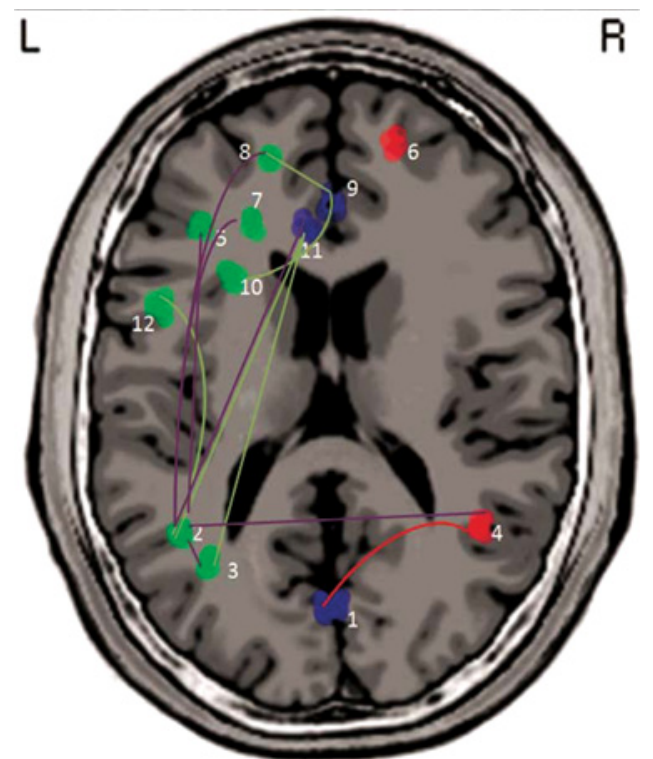

FIGURE 1. sLORETA Functional Connectivity within the default mode network (DMN) for attention deficit/hyperactivity disorder $($ ADHD) $>$ control. Note. The line color represents each frequency domain $($ red $=$ delta; green $=$ alpha- 1 ; purple $=$ alpha- 2 ) There were no significant differences found in theta between groups and beta showed only control $>$ ADHD as seen in Table 2. DMN regions are approximated for 2-D rendering. The lines illustrate the correlations between regions within the DMN and do not represent correlations between frequencies within region. This will be the focus of future works. (Color figure available online.)

\section{fMRI Functional Connectivity}

The analysis of the PPI data collapsed across groups showed a significant interaction between rest/task and the extent to which the medial BA10 time-course predicted activation within the DMN network (Table 3 and Figure 2). This was evident first using the MarsBaR region of interest toolbox, which averages all voxels within an $\mathrm{ROI}$, and then by whole brain analysis

TABLE 3. Results from the PPI Analysis Collapsed Across Group

\begin{tabular}{lllc}
\hline PPI & $\beta$ & $t$ & $p$ \\
\hline Medial BA32 & 0.6 & 1.95 & .038379 \\
Left angular gyrus & 0.67 & 1.86 & .044986 \\
Left BA10 & 0.06 & 0.33 & .373205 \\
Left BA40 & 0.44 & 1.29 & .111562 \\
Left BA7 & 0.72 & 1.62 & .067079 \\
Left BA8 & 0.44 & 1.43 & .090215 \\
Left BA9 & 0.75 & 2.25 & .022993 \\
Right BA40 & 0.61 & 2.55 & .013512 \\
Right BA8_9 & 0.54 & 1.09 & .149865 \\
\hline
\end{tabular}

Note. $\mathrm{PPI}=$ psycho-physiological interaction. with a liberal (uncorrected) threshold. The PPI showed no differences across groups - that is, no difference between ADHD and controls was statistically significant according to rest versus task (Table 4). Next, we examined the random effects model for the noninteracted (raw) BA10 time-course differences between ADHD and control groups (we only examined the cross-group contrast because it is meaningless to look at correlations collapsed across groups, as all regions of the brain are likely to have positive correlations due to correlations in machine noise, e.g., drifts in signal and movement noise, which are reduced by inclusion of movement parameters, prewhitening, high-pass filtering, and detrending, but still remain significant). Medial BA32 and right BA40 showed a significantly greater correlation with the medial BA 10 time-course in controls, whereas the left BA8 showed a subthreshold trend in the same direction (Table 5).

\section{Alpha-Theta Interactions for Precuneal and Prefrontal Regions}

Figure 3 shows results for the correlation analyses of the alpha 1, alpha 2, and theta frequency domains between the anterior cingulate (BA 32 ROI 11), the medial prefrontal (BA 10 ROI 9) and left parietal (BA 39/19 ROI 3) cortex. On the $x$-axis are the ROls by frequency and on the $y$-axis are the Pearson correlation coefficients. In the control group, positive correlations are shown between frequencies, both within and between the ROIs $(p<.05)$. The control group exhibits no inverse correlations between the respective ROls in any frequency. The ADHD group shows no statistically significant correlations. However, there is a trend for a lesser degree of positive associations and an inverse relationship between alpha1 in medial prefrontal and theta, alpha1 and alpha 2 in left parietal. A similar trend for inverse relationship exists between alpha1 and alpha2 in the anterior cingulate and theta and alpha2 in the medial prefrontal region. Correlations shown in the ADHD group reach significance at .05 with rho at .75. This adds support to the lack of contiguity of network nodal cooperation in the DMN during the self-regulation 

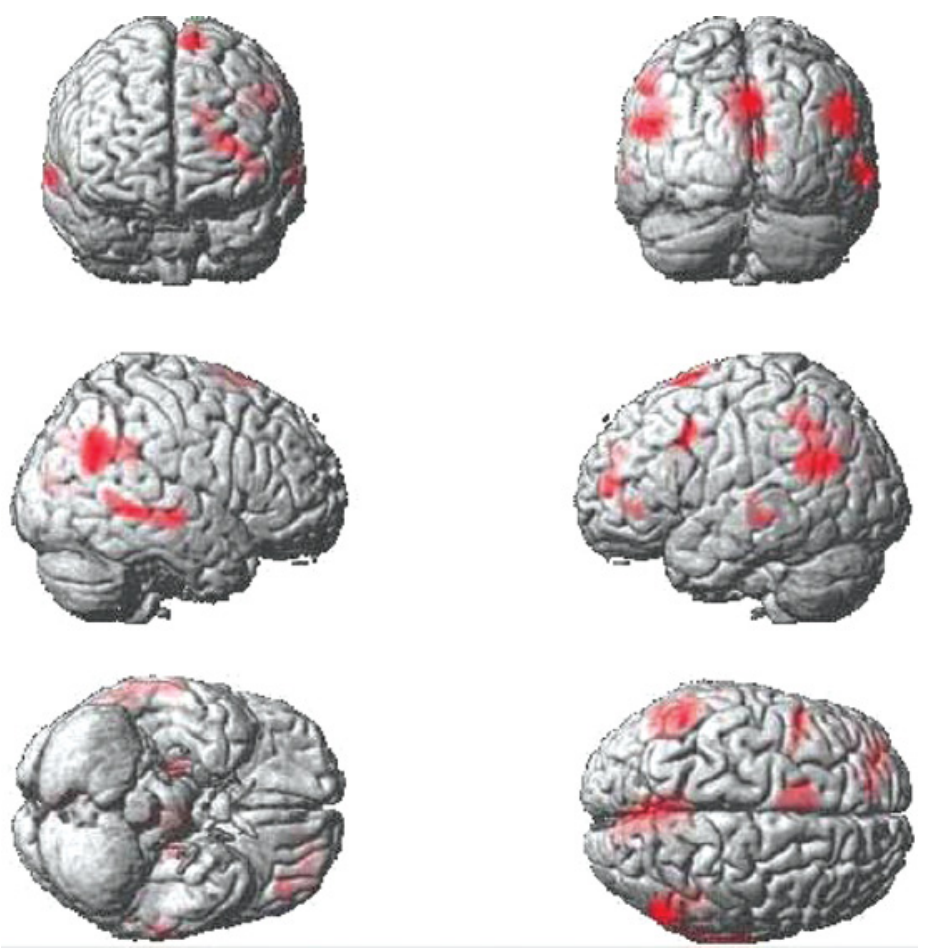

FIGURE 2. Areas from the psycho-physiological interaction analysis collapsed across groups that showed heightened correlations with the medial BA 10 region of interest during rest versus task (uncorrected at $p=.025$ with 100 voxel extent threshold). (Color figure available online.)

TABLE 4. Results from the PPI Analysis Contrasted Across Group

\begin{tabular}{lccc}
\hline PPI & $\beta$ & $t$ & $p$ one-tailed \\
\hline Medial BA32 & -0.3 & -0.96 & .820912 \\
Left angular gyrus & -0.04 & -0.11 & .543088 \\
Left BA10 & 0.08 & 0.42 & .339852 \\
Left BA40 & -0.07 & -0.2 & .577024 \\
Left BA7 & -0.24 & -0.54 & .699021 \\
Left BA8 & -0.2 & -0.64 & .732139 \\
Left BA9 & -0.18 & -0.54 & .699377 \\
Right BA40 & -0.07 & -0.31 & .619159 \\
Right BA8_9 & -0.42 & -0.84 & .790757 \\
\hline
\end{tabular}

Note. PPI = psycho-physiological interaction.

TABLE 5. Results from the Noninteracted Time-Course - Cross Group

\begin{tabular}{lrrr}
\hline PPI & $\beta$ & $t$ & $p$ one-tailed \\
\hline Medial BA32 & 0.39 & 2.44 & .016277 \\
Left angular gyrus & 0.12 & 0.54 & .300508 \\
Left BA10 & 0.05 & 0.59 & .284376 \\
Left BA40 & 0.11 & 0.89 & .196759 \\
Left BA7 & 0.08 & 0.43 & .338357 \\
Left BA8 & 0.13 & 1.48 & .083661 \\
Left BA9 & -0.05 & -0.37 & .640242 \\
Right BA40 & 0.24 & 1.86 & .044851 \\
Right BA8_9 & 0.04 & 0.29 & .387675 \\
\hline
\end{tabular}

Note. PPI = psycho-physiological interaction. associated with baseline even at short distances (Cannon \& Baldwin, 2012).

\section{DISCUSSION}

This is the first study of its kind to examine FC within the DMN using two neuroimaging techniques. This type of analysis may provide important information about intrinsic network integrity and the associated EEG parameters. As suspected, there are significant differences in the functional integrity of the $D M N$ in ADHD as compared to controls, especially for specific frequency domains. Alpha 1 and 2 show significantly greater connectivity between DMN regions in the ADHD group, with a few exceptions between posterior cingulate/precuneus and medial prefrontal and temporal regions. Left parietal, medial prefrontal, and anterior cingulate all show less connectivity as compared to controls. This is an important finding given alpha activity has been shown to decrease during a cross-modal attention task in normal children, but not those with ADHD 


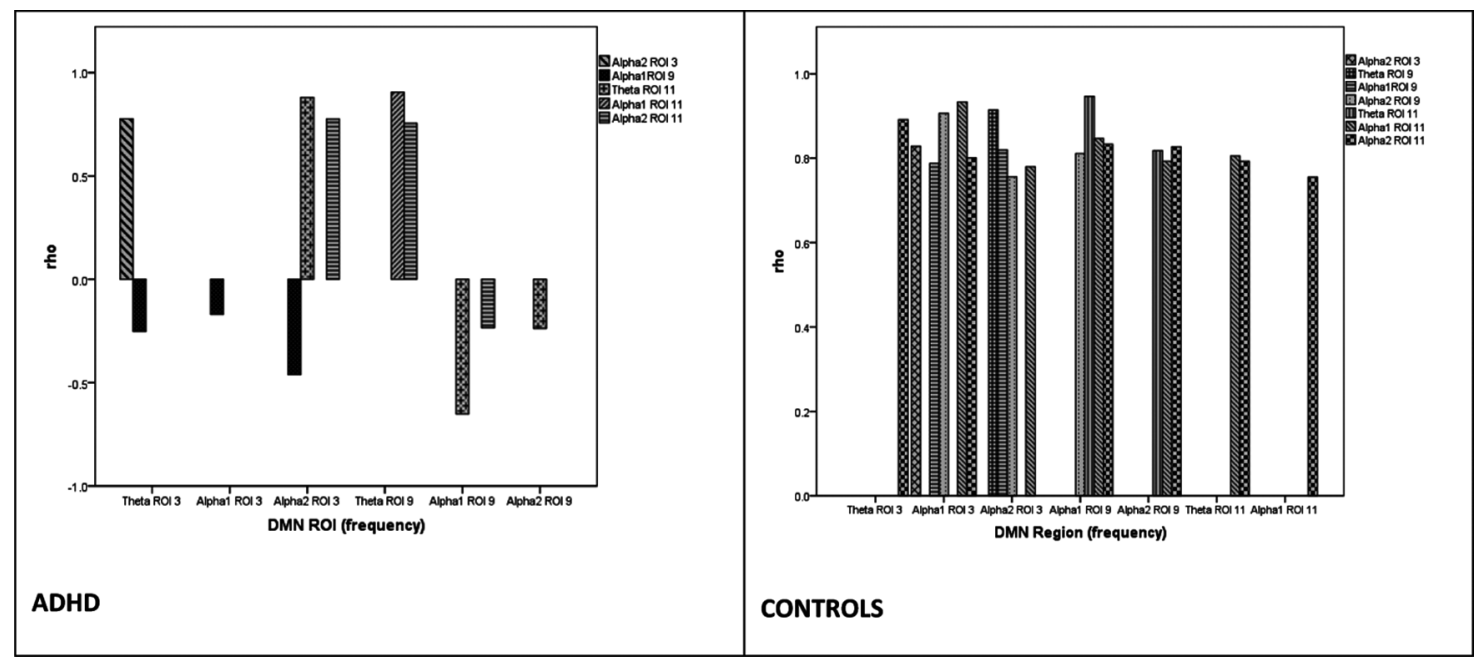

FIGURE 3. Correlations between theta, alpha-1, and alpha-2 frequency domains in 3 default mode network (DMN) regions of interest (ROIs): 11 (BA 32 anterior cingulate), ROI 9 (BA 10 medial prefrontal cortex), and ROI 3 (angular gyrus, parietal lobe). Note. The left graph is for the attention deficit/hyperactivity disorder (ADHD) and the right graph for the control group. Within the graphs are the Pearson correlation coefficient ( $y$-axis) and DMN ROI $\times$ frequency ( $x$-axis). All correlations of the control group are statistically significant at the .05 level. Significance in the ADHD group at $\leq .05$ occurs at .75 .

(Mazaheri et al., 2010). In addition, it was reported that there was an inverse coupling effect for posterior alpha and frontal theta, which may further our understanding of the theta/beta ratio and neural mechanisms involved in the specific deficits characteristic of ADHD (Mazaheri et al., 2010). The results of this pilot study inclusively offer preliminary support to a potential parietal focus (Castellanos et al., 2008) and the interdependency of the alpha and theta frequency domains between parietal and medial prefrontal regions (Mazaheri et al., 2010). It is important to note that the alpha 1 frequency shows greater associations between left parietal and medial prefrontal ROls, the alpha 2 shows greater connectivity between left parietal areas (BA 40 and supramarginal gyrus) and many frontal areas in the ADHD group than in controls, whereas the beta frequency shows the opposite effect (controls > ADHD). This cortical area has been shown to play an important role in the maintenance of executive attention (Cannon et al., 2009). The fMRI functional connectivity adds support to the EEG connectivity, such that higher correlations are found between medial prefrontal and parietal cortices for the control group, including regions shown to be increased in activity by $\mathrm{fMRI}$ and sLORETA for ADHD subjects during baseline as compared to controls (Cannon, Kerson, et al., 2011) and those regions that show positive and negative relationships specific to region and frequency.

Consequently, there would appear to be tentative evidence to support the view that the DMN network is less functionally connected in the ADHD group, but this difference does not interact with the stage (rest vs. active) of the task for the fMRI contrasts. More specifically, we see significant functional connectivity between the DMN ROls in the PPI analysis; furthermore, there are significant differences in that functional connectivity across ADHD versus normal groups. In addition, the fMRI results show that compromised functional integrity of the DMN persists across task and baseline, which is important with respect to specific patterns that can be observed across several spectrums (e.g., ECB, EOB, and task). The EEG CSD features associated with DMN connectivity has remained unexamined until this study. The potential importance of the increased connectivity in alpha between medial prefrontal and parietal regions for the ADHD group may relate to studies that 
propose differential roles for lower $(\sim 8-10 \mathrm{~Hz})$ and upper $(\sim 10-12 \mathrm{~Hz})$ alpha band activity in cognition and attention with the lower band suggested to be primarily involved in response readiness and attentional demands (Klimesch et al., 1992) with an indirect role in memory. By contrast, oscillations in the upper alpha band have been consistently observed during (semantic) long-term memory processes (Klimesch, Doppelmayr, Russegger, \& Pachinger, 1996; Klimesch, Doppelmayr, Russegger, Pachinger, \& Schwaiger, 1998; Klimesch, Schimke, \& Schwaiger, 1994). Thus, these increased levels of alpha band power may provide subtle indications of the characteristic working memory deficits found in ADHD as well as executive, self-regulatory processes (Cannon \& Baldwin, 2012). It has also been reported that LORETA neurofeedback protocols in this left parietal region in the alpha frequency domain produce positive effects in executive functions (Cannon, 2012). This potential is emphasized by data demonstrating that posterior commissural fibers within the corpus callosum have shown the strongest correlation with the alpha frequency, such that the isthmus and Tapetum in the superior occipital cortex show the highest positive association with the alpha frequency. These relationships are suggested to represent cortico-thalamocortical cycles that may modulate the alpha frequency domain. It is interesting that white matter architecture is associated more with the alpha frequency domain than with the neocortical region or grey matter (Valdes-Hernandez et al., 2010). Similar DTI data offer support to the study findings involving posterior regions of the brain (Cao et al., 2010; Hamilton et al., 2008). Studies have proposed the potential role of alpha activity to have an important role in the limbic system and homeostatic and emotional anomalies (limbic irritability) given its associations with several particular syndromes (e.g., depression, anxiety, substance use disorders, and ADHD; Cannon, Baldwin, \& Lubar, 2008; Paquette, Beauregard, \& Beaulieu-Prevost, 2009). In a voxel-based study on regional grey and white matter volumes in 28 male children with
ADHD, grey matter volume deficits were more extensive in the right hemisphere, especially in the prefrontal lobe (BA9, BA10), globus pallidus, and medial parietal cortex (BA7) in ADHD children compared to controls (McAlonan et al., 2007). Similarly, reduced grey matter volume was also measured in the left inferior parietal lobe and superior occipital gyrus. The authors also reported smaller white matter volume in ADHD children that was distributed bilaterally in frontal, temporal, and parietal lobes. The main findings of this study were that, compared to controls, children with ADHD had localized grey matter deficits in predominantly right-sided fronto-parietal brain networks as well as significant volume reduction in associated white matter tracts but no significant differences in whole-brain volume or in total volume of grey or white brain matter or CSF (McAlonan et al., 2007). Of interest, the authors underlined the important role of the cerebellum in the pathogenesis of ADHD, which is in line with the conceptualization that the cerebellum is involved in higher order cognition, including executive function, through intimate reciprocal connections with frontostriatal regions (Leiner, Leiner, \& Dow, 1989; McAlonan et al., 2007; Middleton \& Strick, 1994). The posterior CC and its nearby cortical volumes (e.g., posterior cingulate/precuneus) share extensive connections throughout parietal and occipital cortices, and damage to these regions may be related to decreased volume of the posterior $\mathrm{CC}$, which would be directly associated to decreases in numerous neuropsychological and integrative functions (Cannon, Crane, et al., 2011). These cortical regions and their functional and structural connectivity are a topic for future studies.

The correlations between groups in parietal, medial, and anterior cingulate cortices specific to theta and alpha frequencies offer very interesting insights into network functionality and potential specificity network functionality during baseline condition. The controls show very high positive associations between these three network nodes, whereas the ADHD show quite the opposite with several inverse mechanisms. The important implication among these regions and their associative function lies 
in both self-regulation and executive functions (Suchy, 2009). There is a putative line of thought that the frontal lobes are directly responsible for executive functions, yet this construct remains ambiguous at best, and numerous researchers indicate parietal-frontal networks best account for the concept of executive functions, which may also be better thought of in terms of self-regulation (Cannon \& Baldwin, 2012; Cannon, Baldwin, DiLoreto, \& Phillips, 2012). The fMRI data collapsed across groups for the PPI baseline segments show six areas of significant difference between groups and adds to the validity of the sLORETA results. Clearly the DMN, its functional integrity, and role in self-regulation (executive functions) are important to further our understanding of ADHD and its etiological mechanisms. This is an area of future research for our group.

The current data do have room for improvement. We desire larger samples with additional tasks. The eyes-opened fMRI rest blocks were short relative to the task blocks, and with the small sample size, thresholds of effects are low and sensitivity to false positives may be higher than desired. We may also not be detecting differences in the theta frequency due to the sample size. Nonetheless, the data from both methods do broadly fit with the a priori hypotheses and do form a good direction for a larger scale study. Another limitation is that the EEG data examined the eyes-opened resting baseline and $\mathrm{fMRI}$ examined the resting blocks within the Stroop task. This is an important area for future study given the nature of eyes-opened resting tasks as contrasted with specific cognitive or attentional procedures in the default network. Further study converging either baseline condition or active task would be beneficial and is in the planning process.

In sum, if as suggested by prior research, the alpha frequency is directly associated with attention and working memory, as well as executive functioning, self-regulation and integrative processes (Cannon \& Baldwin, 2012; Cannon, Baldwin, et al., 2011) then what we may be observing are disrupted networks that reflect compromised resources, or more broadly resources devoted to attentional and executive processes are utilized for regulatory purposes in ADHD. For example, in a recent work we evaluated the phenomenology and DMN activity associated with baseline conditions and several active task conditions using sLORETA. The subjective experience of the DMN during baseline consisted of attention, self-regulation, and self-monitoring for task compliance (Cannon \& Baldwin, 2012). The current data provide further insight into the importance of alpha activity to ADHD. This resource-ineffectiveness of alpha in the ADHD brain as such reduces the executive functioning of the individual. Also with increased sample size, significant effects in other frequency domains (viz. theta) may be observed. Several studies indicate alpha to be associated with visual processing, attentional orienting, and cognitive control in normal controls and ADHD (Mazaheri et al., 2010; Zhang, Wang, Bressler, Chen, \& Ding, 2008). In addition, it has been suggested to play an important role in memory; attention; self-perception; and a variety of cognitive, attentional, and affective processes (Cannon, Baldwin, et al., 2008; Cannon, Lubar, Thornton, Wilson, \& Congedo, 2005; Cannon, Lubar, Sokhadze, \& Baldwin, 2008; Sauseng, Klimesch, Gerloff, \& Hummel, 2009; Sauseng et al., 2005). Although the mechanisms of ADHD remain unclear, advancing technologies and interdisciplinary approaches combining methods may aid in developing a less ambiguous understanding of ADHD and its synchronous neural etiology. The current data offer exciting directions for future study across all neuroimaging modalities.

\section{REFERENCES}

Arns, M., Drinkenburg, W., \& Leon Kenemans, J. (2012). The effects of QEEG-informed neurofeedback in ADHD: An open-label pilot study. Applied Psychophysiology and Biofeedback, 37(3), 171-180.

Basser, P. J., Mattiello, J., \& LeBihan, D. (1994a). Estimation of the effective selfdiffusion tensor from the NMR spin echo. Journal of Magnetic Resonance: Series B, 103, 247-254. 
Basser, P. J., Mattiello, J., \& LeBihan, D. (1994b). MR diffusion tensor spectroscopy and imaging. Biophysical Journal, 66, 259267.

Bechtel, N., Kobel, M., Penner, I. K., Klarhofer, M., Scheffler, K., Opwis, K., \& Weber, P. (2009). Decreased fractional anisotropy in the middle cerebellar peduncle in children with epilepsy and/or attention deficit/ hyperactivity disorder: A preliminary study. Epilepsy \& Behavior, 15, 294-298.

Brookes, M. J., Hale, J. R., Zumer, J. M., Stevenson, C. M., Francis, S. T., Barnes, G. R., ... Nagarajan, S. S. (2011). Measuring functional connectivity using MEG: Methodology and comparison with fCMRI. Neuroimage, 56, 1082-1104.

Buckner, R. L., Andrews-Hanna, J. R., \& Schacter, D. L. (2008). The brain's default network: Anatomy, function, and relevance to disease. Annals of the New York Academy of Sciences, 1124, 1-38.

Cannon, R. L. (2012). LORETA neurofeedback: Odd reports, observations, and findings associated with spatial specific neurofeedback training. Journal of Neurotherapy, 16, 164-167.

Cannon, R., \& Baldwin, D. (2012). EEG current source density and the phenomenology of the default network. Clinical Electroencephalography and Neuroscience, 43(4).

Cannon, R., Baldwin, D., DiLoreto, D., \& Khadoumma, A. (2011). Selected abstracts of conference presentations at the 2011 International Society for Neurofeedback and Research (ISNR) 19th ISNR Conference, Phoenix, Arizona. Journal of Neurotherapy, 15, 401-451.

Cannon, R., Baldwin, D., Diloreto, D., \& Phillips, S. (2012). Parietal influences on executive functions and psychopathology: Connecting the dots between self-regulation and psychopathology. Association for Psychological Science 24th annual convention. Chicago, IL.

Cannon, R., Baldwin, D., \& Lubar, J. (2008). Self-perception and experiential schemata in the addicted brain. Applied Psychophysiology and Biofeedback, 33, 223-238.
Cannon, R. L., Baldwin, D. R., Shaw, T. L., Diloreto, D. J., Phillips, S. M., Scruggs, A. M., ... Riehl, T. (2012). Reliability of quantitative EEG (qEEG) measures and LORETA current source density at 30 days. Neuroscience Letters, 518, 27-31.

Cannon, R. L., Congedo, M., Lubar, J. F., \& Hutchens, T. (2009). Differentiating a network of executive attention: LORETA neurofeedback in anterior cingulate and dorsolateral prefrontal cortices. International Journal of Neuroscience, 119, 404-441.

Cannon, R. L., Crane, M. K., Campbell, P. D., Dougherty, J. H., Baldwin, D. R., Effler, J. D., ... Dzloreto, D. (2011). A 9-year-old boy with multifocal encephalomalacia: EEG Loreta and lifespan database, magnetic resonance imaging and neuropsychological agreement. Journal of Neurotherapy, 15, 3-17.

Cannon, R., Kerson, C., \& Hampshire, A. (2011). sLORETA and FMRI detection of medial prefrontal default network anomalies in adult ADHD. Journal of Neurotherapy, 15, 358-373.

Cannon, R. L., Lubar, J. F., Congedo, M., Thornton, K., Towler, K., \& Hutchens, T. (2007). The effects of neurofeedback training in the cognitive division of the anterior cingulate gyrus. International Journal of $\mathrm{Neu}$ roscience, 117, 337-357.

Cannon, R. L., Lubar, J. F., Sokhadze, E., \& Baldwin, D. R. (2008). LORETA neurofeedback for addiction and the possible neurophysiology of psychological processes influenced: A case study and region of interest analysis of loreta neurofeedback in right anterior cingulate cortex. Journal of Neurotherapy, 12, 227-241.

Cannon, R., Lubar, J., Thornton, K., Wilson, S., \& Congedo, M. (2005). Limbic beta activation and LORETA: Can hippocampal and related limbic activity be recorded and changes visualized using LORETA in an affective memory condition? Journal of Neurotherapy, 8, 5-24.

Cao, Q., Sun, L., Gong, G., Lv, Y., Cao, X., Shuai, L., ... Wang, Y. (2010). The macrostructural and microstructural abnormalities 
of corpus callosum in children with attention deficit/hyperactivity disorder: A combined morphometric and diffusion tensor MRI study. Brain Research, 1310, 172-180.

Carmona, S., Vilarroya, O., Bielsa, A., Tremols, V., Soliva, J. C., Rovira, M., ... Bulbena, A. (2005). Global and regional gray matter reductions in ADHD: A voxel-based morphometric study. Neuroscience Letters, 389, 88-93.

Castellanos, F. X., \& Acosta, M. T. (2002). [Syndrome of attention deficit with hyperactivity as the expression of an organic functional disorder]. Revista de Neurologia, 35, 1-11.

Castellanos, F. X., Giedd, J. N., Marsh, W. L., Hamburger, S. D., Vaituzis, A. C., Dickstein, D. P., ... Rapoport, J. L. (1996). Quantitative brain magnetic resonance imaging in attention-deficit hyperactivity disorder. Archives of General Psychiatry, 53, 607-616.

Castellanos, F. X., Lee, P. P., Sharp, W., Jeffries, N. O., Greenstein, D. K., Clasen, L. S., ... Rapoport, J. L. (2002). Developmental trajectories of brain volume abnormalities in children and adolescents with attentiondeficit/hyperactivity disorder. Journal of the American Medical Association, 288, 1740-1748.

Castellanos, F. X., Margulies, D. S., Kelly, C., Uddin, L. Q., Ghaffari, M., Kirsch, A., ... Milham, M. P. (2008). Cingulate-precuneus interactions: A new locus of dysfunction in adult attention-deficit/hyperactivity disorder. Biological Psychiatry, 63, 332-337.

Cavanna, A. E., \& Trimble, M. R. (2006). The precuneus: A review of its functional anatomy and behavioural correlates. Brain, 129(Pt. 3), 564-583.

Coburn, K. L., Lauterbach, E. C., Boutros, N. N., Black, K. J., Arciniegas, D. B., \& Coffey, C. E. (2006). The value of quantitative electroencephalography in clinical psychiatry: A report by the Committee on Research of the American Neuropsychiatric Association. Journal of Neuropsychiatry \& Clinical Neuroscience, 18, 460-500.

Congedo, M., Ozen, C., \& Sherlin, L. (2002). Notes on EEG resampling by natural cubic spline interpolation. Journal of Neurotherapy, 6, 73-80.
Conners, C. K., Erhardt, J. N., Epstein, D., Parker, J. D. A., Sitarenios, G., \& Sparrow, E. (1999). Self-rating of ADHD symptoms in adults I: Factor structure and normative data. Journal of Attention Disorders, 3(3), 141-151.

D’Agati, E., Casarelli, L., Pitzianti, M. B., \& Pasini, A. (2010). Overflow movements and white matter abnormalities in ADHD. Progress in Neuro-psychopharmacology and Biological Psychiatry, 34, 441-445.

Damoiseaux, J. S., Beckmann, C. F., Arigita, E. J., Barkhof, F., Scheltens, P., Stam, C. J., ... Rombouts, S. A. R. B. (2008). Reduced resting-state brain activity in the "default network" in normal aging. Cerebral Cortex, 18, 1856-1864.

Damoiseaux, J. S., Rombouts, S. A., Barkhof, F., Scheltens, P., Stam, C. J., Smith, S. M., ... Beckmann, C. F. (2006). Consistent resting-state networks across healthy subjects. Proceedings of the National Academy of Sciences: U S A, 103, 13848-13853.

Dramsdahl, M., Westerhausen, R., Haavik, J., Hugdahl, K., \& Plessen, K. J. (2012). Adults with attention-deficit/hyperactivity disorderA diffusion-tensor imaging study of the corpus callosum. Psychiatry Research: Neuroimaging, 201, 168-173.

Durston, S., Hulshoff Pol, H. E., Schnack, H. G., Buitelaar, J. K., Steenhuis, M. P., Minderaa, R. B., ... van Engeland, $\mathrm{H}$. (2004). Magnetic resonance imaging of boys with attention-deficit/hyperactivity disorder and their unaffected siblings. Journal of the American Academy of Child and Adolescent Psychiatry, 43, 332-340.

Engel, A. K., Fries, P., \& Singer, W. (2001). Dynamic predictions: Oscillations and synchrony in top-down processing. Nature Reviews Neuroscience, 2, 704-716.

Enzi, B., de Greck, M., Prosch, U., Tempelmann, C., \& Northoff, G. (2009). Is our self nothing but reward? Neuronal overlap and distinction between reward and personal relevance and its relation to human personality. PLoS One, 4, e8429.

Fair, D. A., Cohen, A. L., Dosenbach, N. U., Church, J. A., Miezin, F. M., Barch, D. M., 
... Schlaggar, B. L. (2008). The maturing architecture of the brain's default network. Proceedings of the National Academy of Sciences: U S A, 105, 4028-4032.

Fransson, P., Skiold, B., Horsch, S., Nordell, A., Blennow, M., Lagercrantz, H., \& Aden, U. (2007). Resting-state networks in the infant brain. Proceedings of the National Academy of Sciences: U S A, 104, 15531-15536.

Frei, E., Gamma, A., Pascual-Marqui, R., Lehmann, D., Hell, D., \& Vollenweider, F. X. (2001). Localization of MDMA-induced brain activity in healthy volunteers using low resolution brain electromagnetic tomography (LORETA). Human Brain Mapping, 14, 152-165.

Hamilton, L. S., Levitt, J. G., O’Neill, J., Alger, J. R., Luders, E., Phillips, O. R., ... Nar, K. L. (2008). Reduced white matter integrity in attention-deficit hyperactivity disorder. Neuroreport, 19, 1705-1708.

Hughes, J. R., \& John, E. R. (1999). Conventional and quantitative electroencephalography in psychiatry. Journal of Neuropsychiatry and Clinical Neuroscience, 11, 190-208.

Jasper, H. H. (1958). The ten-twenty electrode system of international federation. Electroencephalography and Clinical Neurophysiology, 10, 371-375.

Klimesch, W., Doppelmayr, M., Russegger, H., \& Pachinger, T. (1996). Theta band power in the human scalp EEG and the encoding of new information. Neuroreport, 7, 12351240.

Klimesch, W., Doppelmayr, M., Russegger, H., Pachinger, T., \& Schwaiger, J. (1998). Induced alpha band power changes in the human EEG and attention. Neuroscience Letters, 244, 73-76.

Klimesch, W., Schimke, H., \& Schwaiger, J. (1994). Episodic and semantic memory: An analysis in the EEG theta and alpha band. Electroencephalography and Clinical Neurophysiology, 91, 428-441.

Leiner, H. C., Leiner, A. L., \& Dow, R. S. (1989). Reappraising the cerebellum: What does the hindbrain contribute to the forebrain? Behavioral Neuroscience, 103, 9981008.
Mazaheri, A., Coffey-Corina, S., Mangun, G. R., Bekker, E. M., Berry, A. S., \& Corbett, B. A. (2010). Functional disconnection of frontal cortex and visual cortex in attentiondeficit/hyperactivity disorder. Biological Psychiatry, 67, 617-623.

McAlonan, G. M., Cheung, V., Cheung, C., Chua, S. E., Murphy, D. G., Suckling, J., ... Ho, T. P. (2007). Mapping brain structure in attention deficit-hyperactivity disorder: A voxel-based MRI study of regional grey and white matter volume. Psychiatry Research: Neuroimaging, 154, 171-180.

Middleton, F. A., \& Strick, P. L. (1994). Anatomical evidence for cerebellar and basal ganglia involvement in higher cognitive function. Science, 266, 458-461.

Northoff, G. (2005). Is emotion regulation self-regulation? Trends in Cognitive Science, 9, 408-409; author reply 409-410.

Northoff, G., \& Bermpohl, F. (2004). Cortical midline structures and the self. Trends in Cognitive Science, 8, 102-107.

Northoff, G., Heinzel, A., de Greck, M., Bermpohl, F., Dobrowolny, H., \& Panksepp, J. (2006). Self-referential processing in our brain-A meta-analysis of imaging studies on the self. Neuroimage, 31, 440-457.

Northoff, G., Schneider, F., Rotte, M., Matthiae, C., Tempelmann, C., Wiebking, C., ... Panksepp, J. (2009). Differential parametric modulation of self-relatedness and emotions in different brain regions. Human Brain Mapping, 30, 369-382.

Ogrim, G., Kropotov, J., \& Hestad, K. (2012). The QEEG theta/beta ratio in ADHD and normal controls: Sensitivity, specificity, and behavioral correlates. Psychiatry Research. http://dx.doi.org/10.1016/j.psychres.2011. 12.041.

Overmeyer, S., Bullmore, E. T., Suckling, J., Simmons, A., Williams, S. C., Santosh, P. J., \& Taylor, E. (2001). Distributed grey and white matter deficits in hyperkinetic disorder: MRI evidence for anatomical abnormality in an attentional network. Psychological Medicine, 31, 1425-1435.

Pascual-Marqui, R. D., Esslen, M., Kochi, K., \& Lehmann, D. (2002). Functional imaging 
with low-resolution brain electromagnetic tomography (LORETA): A review. Methods and Findings in Experimental and Clinical Pharmacology, 24(Suppl C), 91-95.

Pascual-Marqui, R. D., Michel, C. M., \& Lehmann, D. (1994). Low resolution electromagnetic tomography: A new method for localizing electrical activity in the brain. International Journal of Psychophysiology, 18(1), 49-65.

Paquette, V., Beauregard, M., \& BeaulieuPrevost, D. (2009). Effect of a psychoneurotherapy on brain electromagnetic tomography in individuals with major depressive disorder. Psychiatry Research: Neuroimaging, 174(3), 231-239.

Pascual-Marqui, Michel, \& Lehmann, (1994). Pascual-Marqui, Esslen, Kochi, \& Lehmann, 2002.

Pavuluri, M. N., Yang, S., Kamineni, K., Passarotti, A. M., Srinivasan, G., Harral, E. M., ... Zhou, X. J. (2009). Diffusion tensor imaging study of white matter fiber tracts in pediatric bipolar disorder and attentiondeficit/hyperactivity disorder. Biological Psychiatry, 65, 586-593.

Peterson, D. J., Ryan, M., Rimrodt, S. L., Cutting, L. E., Denckla, M. B., Kaufmann, W. E., \& Mahone, E. M. (2011). Increased regional fractional anisotropy in highly screened attention-deficit hyperactivity disorder (ADHD). Journal of Child Neurology, 26, 1296-1302.

Pizzagalli, D. A., Oakes, T. R., \& Davidson, R. J. (2003). Coupling of theta activity and glucose metabolism in the human rostral anterior cingulate cortex: An EEG/PET study of normal and depressed subjects. Psychophysiology, 40, 939-949.

Raichle, M. E., MacLeod, A. M., Snyder, A. Z., Powers, W. J., Gusnard, D. A., \& Shulman, G. L. (2001). A default mode of brain function. Proceedings of the National Academy of Sciences: U S A, 98, 676-682.

Sauseng, P., Klimesch, W., Gerloff, C., \& Hummel, F. C. (2009). Spontaneous locally restricted EEG alpha activity determines cortical excitability in the motor cortex. Neuropsychologia, 47, 284-288.
Sauseng, P., Klimesch, W., Stadler, W., Schabus, M., Doppelmayr, M., Hanslmayr, S., ... Birbaumer, N. (2005). A shift of visual spatial attention is selectively associated with human EEG alpha activity. European Journal of Neuroscience, 22, 2917-2926.

Shulman, G. L., Corbetta, M., Buckner, R. L., Raichle, M. E., Fiez, J. A., Miezin, F. M., \& Petersen, S. E. (1997). Top-down modulation of early sensory cortex. Cerebral Cortex, 7, 193-206.

Shulman, G. L., Ollinger, J. M., Akbudak, E., Conturo, T. E., Snyder, A. Z., Petersen, S. E., \& Corbetta, M. (1999). Areas involved in encoding and applying directional expectations to moving objects. The Journal of Neuroscience, 19, 9480-9496.

Shulman, G. L., Schwarz, J., Miezin, F. M., \& Petersen, S. E. (1998). Effect of motion contrast on human cortical responses to moving stimuli. Journal of Neurophysiology, 79, 2794-2803.

Silk, T. J., Vance, A., Rinehart, N., Bradshaw, J. L., \& Cunnington, R. (2009). Structural development of the basal ganglia in attention deficit hyperactivity disorder: A diffusion tensor imaging study. Psychiatry Research: Neuroimaging, 172, 220-225.

Smith, C. D., Lori, N. F., Akbudak, E., Sorar, E., Gultepe, E., Shimony, J. S., ... Conturo, T. E. (2009). MRI diffusion tensor tracking of a new amygdalo-fusiform and hippocampofusiform pathway system in humans. Journal of Magnetic Resonance Imaging, 29, 12481261.

Suchy, Y. (2009). Executive functioning: overview, assessment, and research issues for non-neuropsychologists. Annals of Behavioral Medicine, 37(2), 106-116.

Sun, L., Cao, Q., Long, X., Sui, M., Cao, X., Zhu, C., ... Wang, Y. (2012). Abnormal functional connectivity between the anterior cingulate and the default mode network in drug-naive boys with attention deficit hyperactivity disorder. Psychiatry Research: Neuroimaging, 201, 120-127.

Takahashi, M., Iwamoto, K., Fukatsu, H., Naganawa, S., lidaka, T., \& Ozaki, N. (2010). White matter microstructure of the 
cingulum and cerebellar peduncle is related to sustained attention and working memory: A diffusion tensor imaging study. Neuroscience Letters, 477, 72-76.

Tian, L., Jiang, T., Liang, M., Zang, Y., He, Y., Sui, M., \& Wang, Y. (2008). Enhanced resting-state brain activities in ADHD patients: A fMRI study. Brain Development, 30, 342-348.

Valdes-Hernandez, P. A., Ojeda-Gonzalez, A., Martinez-Montes, E., Lage-Castellanos, A., Virues-Alba, T., Valdes-Urrutia, L., \& ValdesSosa, P. A. (2010). White matter architecture rather than cortical surface area correlates with the EEG alpha rhythm. Neuroimage, 49, 2328-2339.

van Ewijk, H., Heslenfeld, D. J., Zwiers, M. P., Buitelaar, J. K., \& Oosterlaan, J. (2012). Diffusion tensor imaging in attention deficit/ hyperactivity disorder: A systematic review and meta-analysis. Neuroscience and Biobehavioral Review, 36, 1093-1106.

Weissman, D. H., Roberts, K. C., Visscher, K. M., \& Woldorff, M. G. (2006). The neural bases of momentary lapses in attention. Nature Neuroscience, 9, 971-978.

Wolosin, S. M., Richardson, M. E., Hennessey, J. G., Denckla, M. B., \& Mostofsky, S. H. (2009). Abnormal cerebral cortex structure in children with ADHD. Human Brain Mapping, 30, 175-184.

Zang, Y. F., He, Y., Zhu, C. Z., Cao, Q. J., Sui, M. Q., Liang, M., ... Wang, Y. F. (2007). Altered baseline brain activity in children with ADHD revealed by resting-state functional MRI. Brain Development, 29, 83-91.

Zhang, Y., Wang, X., Bressler, S. L., Chen, Y., \& Ding, M. (2008). Prestimulus cortical activity is correlated with speed of visuomotor processing. Journal of Cognitive Neuroscience, 20, 1915-1925.

Zumsteg, D., Andrade, D. M., \& Wennberg, R. A. (2006). Source localization of small sharp spikes: Low resolution electromagnetic tomography (LORETA) reveals two distinct cortical sources. Clinical Neurophysiology, 117, 1380-1387. 